Aus dem Bergsanatorium (Leitender Arzt: Dr. Th. Janssen) und aus dem Deutschen Kriegerkurhaus (Leitender Arzt: Dr. G. Burkhardt) in Davos.

\section{Zur Frage der Kieselsäuretherapie bei Lungentuberkulose.}

Von Dr. A. Kesseler, 1. Assistent am Sanatorium „Wehrawald“ bei Todtmoos (Schwarzwald).

In den letzten Jahren ist von verschiedenen Seiten, in Deutschland besonders nachdrücklich und eingehend von Kobert'(1), die Behand lung der Lungentuberkulose mit Kieselsäure $\left(\mathrm{SiO}_{2}\right)$ begründet und empfohlen worden. Es wurde auch wiederholt, vor allem von $\mathrm{K}$ üh n (2) und $B$ a u e r (3), über günstige Erfolge mit dieser Therapie berichtet. Infolgedessen wurde in Davos im Bergsanatorium von Herrn Dr. Jans se n und im Deutschen Kriegerkurhaus von Herrn Dr. Burkhardt die Kieselsäure bei Lungentuberkulösen erprobt. lch dankc den Chefärzten beider Häuser die Möglichkeit der Veröffentlichung einer von mir angestellten Nachprüfung des Einflusses der Kieselsäure auf das Leukozytenbild sowie unserer Ansichten und Erfahrungen bezüglich dieses Mittels.

Kobert (1) erblickte eine wesentliche Stütze für seine Empfehlung der Kieselsäure in der von $\mathrm{Schmidt}$ (4) bei Darreichung von Natr. silic. puriss., von $\mathrm{Zickgraf}(5), \mathrm{Sch}$ warz (6), Ladendor und Helwig (7) bei Trinken der Glashäger Quelle, eines im Liter $42,7 \mathrm{mg}$ Metakieselsäure enthaltenden Mineralwassers, übereinstiminend gefundenen Vermehrung der Zahl der Leukozyten, insbesondere der neutrophilen, und Verbesserung des Blutbildes durch, Kernreifung im Sinne Arneths.

Ich habe nun daraufhin im Bergsanatorium. die Wirkung eines auf Dr. Jansse ns Veranlassung hergestellten "Silicium vegetabile, Dialysatum Golaz" mit $43-272 \mathrm{mg} \mathrm{\textrm {SiO } _ { 2 }}$ in der Tagesdosis von dre Teelöffeln bei fünf Fällen und im Kriegerkurhaus bei 11 weiteren Fällen die von $0,5 \mathrm{~g}$ Tabletten von folgender Zusammensetzung: Calc. phospholact. pulv. subt. $\mathrm{kg} 1$, Acid. silic. pulv. subt. 1,5, Calc. phosphor. pulv. subt. 2,5 (also $0,75 \mathrm{mg} \mathrm{SiO}$. pro Tablette) geprüft. Um die physiologischen Tagesschwankungen auszuschalten und die Verdauungsleukozytose $z u$ vermeiden, erfolgte die Blutentnahme frühmorgens am nüchtemen Patienten im Bett. Andere das Blutbild ändernde therapeutische Maßnahmen, wie insbesondere Tuberkulinbehandlung, wurden unterlassen. Bei den Versuchsfällen des Bergsanatoriums wurde zudem die Kieselsäuredarreichung nicht, wie wir dort sonst gewohnt waren und es im allgemeinen für nützlich halten, mit Kalziumgaben kombiniert. Zwei weitere Versuchsfälle wurden außer Betracht gelassen, weil einmal die Entwicklung eines Furunkels, ein andermal das Aufflackern eines alten Mittelohrkatarrhs an der auffälligen Differenz der Leukozytenzahlen hätten Mit- bzw. Hauptursache sein können.

Die Ergebnisse der Blutuntersuchungen sind folgende ${ }^{1}$ ):

Nach der Siliziumeinverleibung haben in vier Fällen die Leukozytenzahlen abgenommen, in einem Fall ist der Unterschied gleich Null, in elf Fällen ist eine Vermehrung erfolgt. Die Durchschnittszahl der Leukozyten ist vor Siliziumgebrauch 9206, nachher 10384 ; die durchschnittliche Zunahme beträgt somit 12,8\% . Die Vermehrung betrifft hauptsächlich die neutrophilen Zellen, erheblich weniger die Lymphozyten. Die absoluten Zahlen beider Zellarten zeigen,

1) Eine genaue tabellarische Uebersicht der Ergebnisse konnte wegen Raummangels nicht cingefigt werden. daß die Neutrophilen mit $78,8 \%$, die Lymphozyten nur mit $92 \%$ an der Gesamtzunahme beteiligt sind. Relativ zur Gesamtzahl der Leukozyten beträgt die Zahl der Neutrophilen vor Siliziumgebrauch $58,8 \%$, nachher $61,10 \%$, die der Lymphozyten $30,3 \%$ bzw. 27,90\%. Relativ haben sich also die Neutrophilen um 2,3\% vermehrt, die Lymphozyten um 2,4\% vermindert. Die Vermehrung der Leukozyten erweist sich von der Höhe der Dosis abhängig und ist nach dem kieselsäurereicheren Dialysat bei den ersten fünf Fällen durchschnittlich viel stärker als nach den Tabletten bei den elf übrigen Fällen.

Die Ergebnisse meiner Untersuchung stimmen mit denen der genannten Voruntersucher in der Hauptsache überein. Die von mir gefundene Vermehrung der Leukozyten ist nur lange nicht so groB und auffallend wie anderwärts, wo sie bis $z u 200 \%$, gegenüber $68.1 \%$ Höchstzunahme in meinem Falle Nr. 3, betrug. Dieser Unterschied dürfte sich zur Genüge erklären lassen aus der geringeren Siliziumdosis bei den meisten meiner Fälle, während die Voruntersucher meist mehr als $30 \mathrm{mg}$ pro Tag gegeben hatten, und aus der Tatsache, $\mathrm{da} \beta$ diese das Blut untertags kurz nach der Kieselsäureverabreichung entnahmen, ich hingegen durchweg mindestens 12 Stunden später. In meinem Falle 3, wo noch 1 Stunde vor der Blutentnahme $28 \mathrm{mg}$ $\mathrm{SiO}_{2}$ genommen waren, ergab sich auch die Höchstzunahme von $68,1 \%$. Zum Ueberfluß könnte für den Unterschied noch der zum Teil durch die Auswahl meiner Fälle bedingte, an sich bereits hohe Anfangsleukozytenstand verantwortlich gemacht werden, der eine gewisse Erschöpfung der leukozytären Bildungsstätten verursacht haben mag. Die beiden ersten Gründe möchte ich auch dafür anführen, daB in meiner Untersuchung die Verschiebung des Blutbildes nach rechts durch Kernreifung im Sinne A rneths nicht so auffallend erscheint, wenn aucl einzelne Fälle, wie $\mathrm{Nr} .1$ und $\mathrm{Nr}$. 16, darauf hindeuten. Als neu möchte ich aus meiner Untersuchung die Feststellung hervorheben, da $\beta$ die Siliziumleukozytose. wenn auch bereits vermindert, doch noch nach 12 Stunden nachweisbar ist.

Es erhebt sich nun die Frage, welcher Nutzen von einer Leukozytose, speziell der Vermehrung der neutrophilen Zellen, für die Heilung eines tuberkulösen Prozesses zu erwarten ist. Aus der Literatur: Sahli (8), Schenitzky (9), Weill (10) ergibt sich, daB bei initialer leichter Tuberkulose der Lungen eine Lymphozytose, bei fortgeschrittener schwerer eine Vermehrung der Neutrophilen die Regel ist. Baer und Engels man (11) glauben, in der durch Hochgebirgsaufenthalt hervorgerufenen Lymphozytose eine günstige Wirkung des Hochgebirges erblicken zu dürfen, und nehmen nach ihren eigenen und $\mathrm{S}$ tef $\mathrm{fe} \mathrm{n}$ s gleichen Untersuchungsergebmissen und gestützt auf Bartels, Neumanns, Schroeders und Webbs Nachweis einer Virulenzschädigung der Tuberkelbazillen durch Lymphdrüsen- und Milzextrakt an, daß ,unter den weißen Blutzellen den Lymphozyten im Kampfe des Organismus mit der Tuberkulose die Prävalenz gebühre". Für diese Ansicht spricht auch der nach $M$ as. s u r (12) und anderen günstige Einfluß einer Schilddrüsenvergrößerung auf den Verlauf der Lungentuberkulose; denn auch Schilddrüsenvergrößerung geht meistens mit Lymphozytose einher. Die infolge von Jodanwendung, nach subkutanen Jodoforminjektionen und nach Jodanstrich insbesondere von $\mathrm{Hotz}$ (13) nachgewiesene, Lymphozytose veranlaßt Rothschild und Soden (14), zu behaupten, die beste Chemotherapie der Tuberkulose beruhe zur Zeit auf Jodanwendung, Jod rufe Lymphozvtose hervor, und „ohne Lymphozytose keine Heilung der Tuberkulose"s.

Wenn danach die Erzielung einer Lymphozytose bei der Behandlung Tuberkulöser zweckmäßiger erscheint, so ist doch auch von einer Vermehrung der mehrkernigen weißen Blutzellen eine heilsame Einwirkung auf den tuberkulösen Proze $B$ zu erwarten, sofern das die Leukozy tose anregende Agens den Organismus nicht sonst irgendwie schädigt. Zunächst kann eine solche neutrophile Leukozytose, wie die des Siliziums, nützen, indem sie die physiologische Verdauungsleukozytose steigert und dadurch den Abbau, Aufbau und die Assimilation der Nahrung, besonders der Eiweißkörper, unterstützt. Dann ist man gewohnt, vor allem in den neutrophilen Leukozyten die Schutztruppen des Organismus zu sehen, die einerseits durch besondere Beteiligung an der Antitoxinbildung zur Bindung und Zerstörung von Giften beitragen und anderseits als Phagozvten, sei es durch Aufnahme lebender oder nur toter Bakterien, wichtige Polizei- bzw. Straßenkehrdienste leisten. Eine erhöhte Phagozytose als Siliziumwirkung ist ja auch von $\mathrm{Helwig}$ (7) nachgewiesen worden. Ebenso pflegt man bei anderen im Kampfe gegen die Tuberkulose geschätzten Heilmitteln, zum Beispiel bei der Zimtsäure, dem Hetol. denı Elbon, deren leukozytoseerregenden Kraft große Bedeutung beizumessen. Und die spezifische Tuberkulintherapie geht meist mit Vermehrung der neutrophilen Blutzellen einher, Sahli (8), Schenitzky (9) u. a. Die Siliziumleukozytose scheint schließlich noch durch Marys (15) Untersuchungen an Wichtigkeit zu gewinnen. Mary vermißte bei Tuberkulose im Blutserum gewisse ultramikroskopische Körnchen, Zerfallsprodukte neutrophiler Myelozyten. Er erblickt darin die Folge einer durch Demineralisation hervorgerufenen Unzulänglichkeit des Knochenmarkes, die er durch Phosphor- und Siliziumgaben beheben zu können glaubt.

So sind wir schon auf Grund der Resultate der Blutuntersuchung reneigt, das Silizium in der Phthisiotherapie weiter zu empfehlen. Allein auch klinisch glauben wir durch monatelange Kieselsäurezufuhr eine günstige Beeinflussung des Krankheitsbildes gesehen zu haben; wenn uns auch nicht wie $K u ̈ h n$ (2) der Krankheitsverlauf unter diese Therapie ganz anders als gewohnt erschien. In einem Falle, der ein halbes Jahr lang täglich regelmäBig Silizium als Tee und Dialysat 
bekommen hatte und der nach Suizid zur Autopsie kam, stellten wir eine selbst fürs Hochgebirge überraschend starke und ausgedehnte bindegewebige Umwandlung und Abkapselung der zahlreichen kranken Lungenherde fest. Dieser Fall erscheint geeignet, am Menschen das Ergebnis der Meerschweinchenversuche $\mathrm{Kahles}$ (16) und $\mathrm{Röss-}$ le 8 (17) zu bestätigen. Die beiden Autoren konnten bei tuberkuloseinfizierten Tieren aus dem Grade der fibrösen und indurierenden Herdveränderungen die genaue Diagnose stellen, welche Tiere sie mit Silizium behandelt hatten und welche nicht.

Es wäre noch die Frage zu beantworten, in welcher Form das Silizium verabreicht werden soll. Im Bergsanatorium wurde anfänglich lange Zeit ein Tee nach $K \ddot{u} h \mathrm{n} s$ Vorschrift:

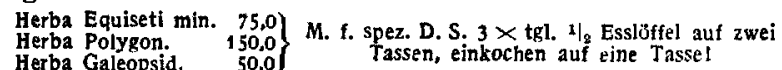

bereitet und gegeben. $\mathrm{Da}$ die Zubereitung sehr umständlich und kostspielig war und Patienten der Praxis auBerhalb des Sanatoriums kaum, längere Zeit zugemutet werden kann, hat Herr Dr. Ja ns se n die Herstellung des schon genannten Dialysates veranlaßit, welches der obigen Kombination von $K$ üh $n$ genau entspricht und den gleichen zwischen 43 und $272 \mathrm{mg}$ schwankenden Siliziumgehalt hat, wie der Tee in der Tagesdosis von drei Tassen in der von drei Teelöffeln aufweisen soll. Der nicht gerade angenehme Geschmack des Tees sowoh wie des Dialysates kann durch Korrigentien, wie z. B. Succus liquiritiae, verdeckt werden. Beide Medikationen wurden jedoch auch ohnedies auf Verordnung regelmäBig und willig von der Mehrzahl aller wegen Tuberkulose behandelter Patienten genommen. Ueber Störung des Appetits, Magenbeschwerden oder sonstige Schädigung wurde selbst nach doppelten Dosen davon nicht geklagt. - Im Kriegerkurhaus hat man, auf sparsamere Arzneiverordnung angewiesen, da ani reine Natrium-silic.-Tabletten allzuoft dyspeptische Beschwerden entstanden, von der Ansicht ausgehend, daB eine Kombination mit Kalzium nützlich sei und homöopathischere Dosen das Aufspeicherungsvermögen des Organismus erschöpfen, die Tabletten von der obengenannten Zusammensetzung angewandt. In Wasser zerfallen und teilweise gelöst, schmecken sie leicht säuerlich, sicher nicht unangenehm, und waren niemals nachweislich an Appetit- und Magenstörung schuldig. -

Wo ein siliziumhaltiges Mineralwasser, wie die angeblich wohlschmeckende Glashäger Quelle, zur Verfügung steht, kann ein solches mit Vorteil verordnet werden. Im Bergsanatorium wurde in letzter Zeit der von Liebreich empfohlene Lamscheider Stahlbrunnen erprobt, der im Liter $31,7 \mathrm{mg}$ Kieselsäure enthält und besonders bei anämischen Tuberkulösen zwei verschiedenen Indikationen genügen kann, da sich bei den bisherigen Versuchen als Eisenwirkung eine deutliche Zunahme des Hämoglobingehaltes und der Erythrozytenzahl ergeben hat. Als billigerer und bequemerer, für ausgedehnte Anwendung geeigneterer Ersatz eines natürlichen Siliziumwassers käme ein Präparat in Salz- oder Komprettenform in Betracht, das durch Eindampfen eines guten Brunnens gewonnen wird oder dessen Analyse entsprechend künstlich zusammengesetzt ist. Selbstverständlich verdienen in der Phthisiotherapie auch schon früher beliebte Heilund Kräftigungsmittel, die sich, wie z. B. Malzextrakt, durch Kieselsäurereichtum auszeichnen, besondere Beachtung. Als ganz unerläßlich erscheint es jedoch, bei der Wahl und Zubereitung der Speisen auf deren $\mathrm{SiO}_{2}$-Gehalt Bedacht zu nehmen.

Mit dieser letzten Verabreichungsmöglichkeit wird man auch in Würdigung der hohen Bedeutung, die neben anderen anorganischen Salzen der Kieselsäure für die Erhaltung der Volksgesundheit mehr und mehr beigemessen wird, die wichtige Aufgabe der Prophylaxe am besten erfüllen können. Unter anderen hat Je ss en (18) wieder neuerdings die Ansicht vertreten, die Demineralisation des Organismus sei nicht nur, wie die französische Schule unter Robin gelehrt hat, eine Folge der Tuberkulose, sondern umgekehrt sei die Demineralisation des menschlichen Körpers - hervorgerufen durch die quantitativ und qualitativ verschlechterte Lebensmittelversorgung - Mitursache an der erschreckenden seuchenhaften Ausbreitung dieser Krankheit in den kriegsverarmten Ländern. Der Forderung, die allgemeine Demineralisation auch durch Kieselsäurezufuhr zu beheben und zu verhüten und dadurch zur Prophylaxe der Tuberkulose beizutragen, müssen vor allem wir deutschen Aerzte in eruster Erwägung mit Energie und Ausdauer gerecht werden. Hierfür geeignete wohl. feile und angenehme GenuBmittel, wie in früheren Zeiten 2 . B. malzreiches Kraftbier, stehen heute der Allgemeinheit Zeiten z. B. malzZwangsweise Kieselgur mit im Brot zu verbacken, wie es früher bei Teuerung und Hungersnot, wohl weniger in der richtigen Erkenntnis des der MaBnahme innewohnenden Wertes, als um das Mehl zut strecken, geschah, wird sich auch nicht durchführen lassen. Also bleibt uns nichts anderes übrig, als dem Volke immer wieder ein sparsames Wirtschaften mit dem verringerten Kieselsäuregehalt der Nahrung zu predigen, es, wo angängig, zum Genuß roher, nicht durch Kochen entmineralisierter Speisen zu mahnen und dem heutigentags als Unfug anzusehenden Wegschütten des Abkochwassers vom Gemüse allüberall $\mathrm{zu}$ steuern.

Litera tur: 1. Kobert, Ueber Kieselsaurehaltige Heilmittel, insonderheit bei Tuberkulose. Rostock 1918. - 2. M. m. W. 1918 Nr. 65. - 3. Beitr. z. Klin. d. Tbc. 391918 H. 2. 8. Kib. Bef Ke H. 4. - 11. D. Arch f rlin 1121913 . exper. Path. 191917 - 10. Zcchr. f. Tbc. 29 13. Mitt. Grenzerb. usw. 25 H. M. 112 1913. - 12. Beitr. z. Klin. d. Tbc. 391918 H. 1. Valcenzia, Dezember 1914. 25 H. $16 .-44 . \mathrm{D} . \mathrm{m}$. W. 1913 Nr. 25. - 15 . Rev. de Hie. y. Tub. Deutschlands usw., Stuttgart 1919 . u. 